\title{
The monotonicity and convexity of a function involving psi function with applications
}

\author{
Bang-Cheng Sun ${ }^{1,2}$, Zhi-Ming Liu', Qiang Li ${ }^{1 *}$ and Shen-Zhou Zheng ${ }^{3 *}$
}

\footnotetext{
"Correspondence: qli3@bjtu.edu.cn; shzhzheng@bjtu.edu.cn

'School of Mechanical, Electronic and Control Engineering, Beijing Jiaotong University, Beijing, 100044 China

${ }^{3}$ Department of Mathematics, Beijing Jiaotong University, Beijing, 100044, China

Full list of author information is available at the end of the article
}

\begin{abstract}
In this paper, we prove that the function$$
x \mapsto \exp \left(\psi\left(x+\frac{1}{2}\right)-\frac{1}{24} \frac{1}{x^{2}+7 / 40}\right)-x
$$

is decreasing from $(-1 / 2, \infty)$ onto $(0,1 / 2)$ and convex on $(-1 / 2, \infty)$. As a consequence of the main theorem, various type of bounds for the psi function are presented, which essentially generalize or improve some known results.
\end{abstract}

MSC: Primary 33B15; 26A48; secondary 11B83; 26D15

Keywords: psi function; harmonic number; inequality

\section{Introduction}

For $x>0$, the classical Euler gamma function $\Gamma$ and psi (digamma) function $\psi$ are defined by

$$
\Gamma(x)=\int_{0}^{\infty} t^{x-1} e^{-t} d t, \quad \psi(x)=\frac{\Gamma^{\prime}(x)}{\Gamma(x)},
$$

respectively. Furthermore, the derivatives $\psi^{\prime}, \psi^{\prime \prime}, \psi^{\prime \prime \prime}, \ldots$ are known as polygamma functions.

As an important role played in many branches, such as mathematical physics, probability, statistics, and engineering, the gamma and polygamma functions have attracted the attention of many scholars. Recently, many authors showed numerous interesting inequalities for the digamma (psi) function $\psi$ and the Euler constant defined by

$$
\gamma=\lim _{n \rightarrow \infty}\left(\sum_{k=1}^{n} \frac{1}{k}-\ln n\right)=0.577215664 \cdots,
$$

where $\sum_{k=1}^{n} \frac{1}{k}:=H_{n}$ is called the harmonic number. In particular, there has many approximation formulas for psi function and harmonic number, which can be found in [1-18], and closely related references therein.

(c) 2016 Sun et al. This article is distributed under the terms of the Creative Commons Attribution 4.0 International License (http://creativecommons.org/licenses/by/4.0/), which permits unrestricted use, distribution, and reproduction in any medium, provided you give appropriate credit to the original author(s) and the source, provide a link to the Creative Commons license, and indicate if changes were made. 
We would like to mention DeTemple and Wang's paper [19] for half-integer approximation formulas

$$
\gamma+\ln \left(n+\frac{1}{2}\right)+\frac{1}{24(n+1)^{2}}<H_{n}<\gamma+\ln \left(n+\frac{1}{2}\right)+\frac{1}{24 n^{2}}
$$

and

$$
\gamma+\ln \left(n+\frac{1}{2}\right)+\frac{1}{24 n^{2}}+\frac{7}{960} \frac{1}{(n+1)^{4}}<H_{n}<\gamma+\ln \left(n+\frac{1}{2}\right)+\frac{1}{24 n^{2}}+\frac{7}{960} \frac{1}{n^{4}} .
$$

It was also proved in $[13,20],[21]$, Lemma 1.7 , that

$$
\ln \left(x+\frac{1}{2}\right)<\psi(x+1) \leq \ln \left(x+e^{-\gamma}\right)
$$

for $x>0$, where $\frac{1}{2}$ and $e^{-\gamma}$ are the best possible constants, and $\gamma=0.577215664 \cdots$ is the Euler-Mascheroni constant. Thanks to formula (1.4) and the relation $H_{n}=\gamma+\psi(n+1)$, we have

$$
\gamma+\ln \left(n+\frac{1}{2}\right)<H_{n} \leq \gamma+\ln \left(n+e^{1-\gamma}-1\right)
$$

for any $n \in \mathbb{N}$. In 2011, Batir [22] further proved that

$$
\frac{1}{2} \ln \left(x^{2}+x+e^{-2 \gamma}\right) \leq \psi(x+1)<\frac{1}{2} \ln \left(x^{2}+x+\frac{1}{3}\right)
$$

for all $x>0$, where $e^{-2 \gamma}$ and $1 / 3$ are the best possible. As a direct consequence, he showed that, for $n \in \mathbb{N}$,

$$
\gamma+\frac{1}{2} \ln \left(n^{2}+n+e^{2-2 \gamma}-2\right) \leq H_{n}<\gamma+\frac{1}{2} \ln \left(n^{2}+n+\frac{1}{3}\right) .
$$

Batir $[23,24]$ provided another interesting bound for the psi function:

$$
a-\ln \left(e^{1 / x}-1\right)<\psi(x)<b-\ln \left(e^{1 / x}-1\right),
$$

where $x>0, a \leq \ln 2$, and $b \geq 0$. Consequently, the double inequality

$$
\ln \frac{\pi^{2}}{6}-\ln \left(e^{1 /(n+1)}-1\right)<H_{n}<\gamma-\ln \left(e^{1 /(n+1)}-1\right)
$$

for all $n \in \mathbb{N}$ was attained in Corollary 2.2 in [23]. Later, this inequality was sharpened to

$$
1+\ln (\sqrt{e}-1)-\ln \left(e^{1 /(n+1)}-1\right)<H_{n}<\gamma-\ln \left(e^{1 /(n+1)}-1\right)
$$

for all $n \in \mathbb{N}$ by Alzer [25].

For reader's convenience, here we name this class of bounds for the psi function and harmonic numbers the Batir-type bounds and call the corresponding inequality a Batirtype inequality. 
Also, inequalities (1.10) are equivalent to

$$
a \leq e^{H_{n+1}}-e^{H_{n}}<b
$$

with the best constants $a=e(\sqrt{e}-1) \approx 1.7634$ and $b=e^{\gamma} \approx 1.7810$ (see also [26]). For a more general result, see [3], Theorem 1.3. Similarly, in the context, we call inequalities (1.11) the Alzer-type ones.

Batir [22] further proved some new Batir-type inequalities for the psi functions and harmonic number, in particular,

$$
\frac{1}{2} \ln \frac{2 x+b}{e^{2 /(x+1)}-1} \leq \psi(x+1) \leq \frac{1}{2} \ln \frac{2 x+b}{e^{2 /(x+1)}-1} \quad \text { for } x \geq 0
$$

with the best constants $a=2$ and $b=e^{-2 \gamma}\left(e^{2}-1\right)$. This implies that, for $n \in \mathbb{N}$, we have

$$
\frac{1}{2} \ln \frac{2 n+b}{e^{2 /(n+1)}-1} \leq H_{n} \leq \frac{1}{2} \ln \frac{2 n+b}{e^{2 /(n+1)}-1}
$$

where $a=2$ and $b=e^{2-2 \gamma}(e-1)-2 \approx 2.0024$ are the best possible. Obviously, it is equivalent to the double inequalities

$$
e^{2 \gamma}(2 n+2)<e^{2 H_{n+1}}-e^{2 H_{n}} \leq e^{2 \gamma}(2 n+2.0024 \cdots) .
$$

Clearly, it is an Alzer-type inequality.

On the other hand, Villarino [27], Theorem 1.7, proved that the sequence

$$
d_{n}=\frac{1}{\psi(n+1)-\ln (n+1 / 2)}-\frac{1}{24}\left(n+\frac{1}{2}\right)^{2}
$$

is increasing for $n \in \mathbb{N}$, and meanwhile DeTemple and Wang [19] by an approximation argument for the harmonic number showed, for $n \in \mathbb{N}$, the following inequality:

$$
\frac{1}{24(n+1 / 2)^{2}+21 / 5}<H_{n}-\ln \left(n+\frac{1}{2}\right)-\gamma<\frac{1}{24(n+1 / 2)^{2}+1 /(1-\ln 3+\ln 2-\gamma)-54}
$$

with the best constants $\frac{21}{5}$ and $1 /(1-\ln 3+\ln 2+\psi(1))-54 \approx 3.7393$. Yang et al. [28], Theorem 2, further showed that the function

$$
x \mapsto F_{a}(x)=24\left(x^{2}+a\right)[\psi(x+1 / 2)-\ln x]-1
$$

is strictly completely monotonic on $(0, \infty)$ if and only if $a \geq 7 / 40$.

Motivated by all these recent papers, the aim of this paper is to investigate the monotonicity and convexity of the function related to the psi function and present some new general, Batir-type, and Alzer-type inequalities for the psi function and harmonic number.

\section{Preliminaries}

In this section, let us recall a few of involving lemmas and some basic facts. 
Lemma 1 ([29], pp.258-260) Let $x>0$ and $n \in \mathbb{N}$. Then

$$
\psi^{(n)}(x+1)-\psi^{(n)}(x)=\frac{(-1)^{n} n !}{x^{n+1}} .
$$

Lemma 2 ([29], pp.258-260) As $x \rightarrow \infty$, we have

$$
\begin{aligned}
& \psi(x) \sim \ln x-\frac{1}{2 x}-\frac{1}{12 x^{2}}+\frac{1}{120 x^{4}}-\frac{1}{252 x^{6}}, \\
& \psi^{\prime}(x) \sim \frac{1}{x}+\frac{1}{2 x^{2}}+\frac{1}{6 x^{3}}-\frac{1}{30 x^{5}}+\frac{1}{42 x^{7}} \\
& \psi^{\prime \prime}(x) \sim-\frac{1}{x^{2}}-\frac{1}{x^{3}}-\frac{1}{2 x^{4}}+\frac{1}{6 x^{6}}-\frac{1}{6 x^{8}} .
\end{aligned}
$$

Lemma 3 ([30]) Let $f$ be a function on an interval I such that $\lim _{x \rightarrow \infty} f(x)=0$. If $f(x+1)$ $f(x)>0$ for all $x \in(a, \infty)$, then $f(x)<0$. Conversely, if $f(x+1)-f(x)<0$ for all $x \in(a, \infty)$, then $f(x)>0$.

Lemma 4 ([31], Lemma 7) For $n \in \mathbb{N}$ and $m \in \mathbb{N} \cup\{0\}$ with $n>m$, let $P_{n}(t)$ be a polynomial with $n$ degrees defined by

$$
P_{n}(t)=\sum_{i=m+1}^{n} a_{i} t^{i}-\sum_{i=0}^{m} a_{i} t^{i}
$$

where $a_{n}, a_{m}>0$ and $a_{i} \geq 0$ for $0 \leq i \leq n-1$ with $i \neq m$. Then there exists an unique number $t_{m+1} \in(0, \infty)$ satisfying $P_{n}(t)=0$ such that $P_{n}(t)<0$ for $t \in\left(0, t_{m+1}\right)$ and $P_{n}(t)>0$ for $t \in$ $\left(t_{m+1}, \infty\right)$.

Lemma 5 Let $u$ be the function on $(-\infty, \infty)$ defined by

$$
u(x)=\frac{5}{120 x^{2}+21} .
$$

Then, for $x \neq-1 / 2$, we have

$$
p(x)=-\frac{1}{(x+1 / 2)^{2}}-u^{\prime}(x+1)+u^{\prime}(x)<0 .
$$

Proof Differentiation leads to

$$
u^{\prime}(x)=-\frac{400}{3} \frac{x}{\left(40 x^{2}+7\right)^{2}} .
$$

Factoring gives

$$
\begin{aligned}
p(x) & =-\frac{1}{(x+1 / 2)^{2}}+\frac{400}{3} \frac{x+1}{\left(40(x+1)^{2}+7\right)^{2}}-\frac{400}{3} \frac{x}{\left(40 x^{2}+7\right)^{2}} \\
& =-\frac{1}{(x+1 / 2)^{2}}-\frac{400}{3} \frac{4,800 x^{4}+9,600 x^{3}+6,960 x^{2}+2,160 x-49}{\left(40(x+1)^{2}+7\right)^{2}\left(40 x^{2}+7\right)^{2}} .
\end{aligned}
$$


Replacing $x$ by $(t-1 / 2)$ and factoring, we get

$$
p(x)=-\frac{7,680,000 t^{8}-384,000 t^{6}+2,851,200 t^{4}-531,760 t^{2}+250,563}{3 t^{2}\left(40(t-1 / 2)^{2}+7\right)^{2}\left(40(t+1 / 2)^{2}+7\right)^{2}},
$$

where $t=x+1 / 2$.

Note that the numerator of this fraction can be written as

$$
\begin{aligned}
p_{1}(t) & =7,680,000 t^{8}-384,000 t^{6}+2,851,200 t^{4}-531,760 t^{2}+250,563 \\
& =4,800 t^{4}\left(40 t^{2}-1\right)^{2}+\frac{7,692,800}{3} t^{4}+\frac{1}{3}\left(920 t^{2}-867\right)^{2}>0,
\end{aligned}
$$

and the desired result easily follows.

Lemma 6 Let $u$ and $p$ be defined by (2.6) and (2.7). Suppose that $q$ and $r$ are defined on $(-1 / 2, \infty)$ by

$$
\begin{aligned}
& q(x)=\frac{2}{(x+1 / 2)^{3}}-u^{\prime \prime}(x+1)+u^{\prime \prime}(x), \\
& r(x)=-\frac{1}{(x+1 / 2)^{2}}-u^{\prime}(x+1)-u^{\prime}(x) .
\end{aligned}
$$

Then, for $x>-1 / 2$, we have

$$
S(x):=-\frac{2}{(x+1 / 2)^{2}}+r(x+1)+\frac{q(x+1)}{p(x+1)}-r(x)-\frac{q(x)}{p(x)}<0 .
$$

Proof An immediate computation yields

$$
u^{\prime \prime}(x)=\frac{400}{3} \frac{120 x^{2}-7}{\left(40 x^{2}+7\right)^{3}} .
$$

Then we get

$$
\begin{aligned}
& q(x)=\frac{2}{(x+1 / 2)^{3}}-\frac{400}{3} \frac{120(x+1)^{2}-7}{\left(40(x+1)^{2}+7\right)^{3}}+\frac{400}{3} \frac{120 x^{2}-7}{\left(40 x^{2}+7\right)^{3}}, \\
& r(x)=-\frac{1}{(x+1 / 2)^{2}}+\frac{400}{3} \frac{x+1}{\left(40(x+1)^{2}+7\right)^{2}}+\frac{400}{3} \frac{x}{\left(40 x^{2}+7\right)^{2}} .
\end{aligned}
$$

Substituting $p(x), q(x), r(x)$ into $S(x)$ and factoring it give

$$
S(x)=-\frac{16 \times 10^{11}}{3} \frac{S_{2}(x)}{S_{1}(x)}
$$

where

$$
\begin{aligned}
S_{1}(x)= & (2 x+1)^{2}(2 x+3)^{2}\left(40 x^{2}+7\right)^{2}\left(40 x^{2}+160 x+167\right)^{2} \\
& \times\left(7,680,000 x^{8}+92,160,000 x^{7}+483,456,000 x^{6}+1,448,064,000 x^{5}\right. \\
& +2,711,491,200 x^{4}+3,257,107,200 x^{3}+2,458,239,440 x^{2}
\end{aligned}
$$




$$
\begin{aligned}
& +1,069,159,920 x+205,944,303) \\
& \times\left(7,680,000 x^{8}+30,720,000 x^{7}+53,376,000 x^{6}+52,608,000 x^{5}\right. \\
& +35,011,200 x^{4}+18,182,400 x^{3}+6,745,040 x^{2} \\
& +1,301,840 x+319,823) \\
& S_{2}(x)=19,544,408,064 x^{20}+390,888,161,280 x^{19}+\frac{18,277,115,756,544}{5} x^{18} \\
& +\frac{106,181,831,688,192}{5} x^{17}+\frac{2,147,345,768,669,184}{25} x^{16} \\
& +\frac{6,422,868,775,010,304}{25} x^{15}+\frac{368,287,175,087,671,296}{625} x^{14} \\
& +\frac{662,967,302,010,630,144}{625} x^{13}+\frac{4,756,138,453,310,742,528}{3,125} x^{12} \\
& +\frac{5,496,272,101,145,296,896}{3,125} x^{11}+\frac{25,764,390,625,415,987,616}{15,625} x^{10} \\
& +\frac{3,939,903,200,496,190,272}{3,125} x^{9}+\frac{12,333,289,847,706,921,772}{15,625} x^{8} \\
& +\frac{6,339,553,647,515,390,816}{15,625} x^{7}+\frac{26,760,964,338,980,254,467}{156,250} x^{6} \\
& +\frac{4,616,788,558,072,176,841}{78,125} x^{5}+\frac{513,733,037,814,725,250,509}{31,250,000} x^{4} \\
& +\frac{27,954,545,230,825,852,509}{7,812,500} x^{3}+\frac{44,860,757,315,321,422,071}{78,125,000} x^{2} \\
& +\frac{2,404,936,823,928,444,981}{39,062,500} x+\frac{389,355,305,888,516,211,027}{100,000,000,000} \text {. }
\end{aligned}
$$

We further prove that $S_{1}(x), S_{2}(x)>0$ for $x>-1 / 2$. In fact, replacing $x$ by $(t-1 / 2)$ in $(2.14)$ and arranging, we obtain

$$
\begin{aligned}
S_{1}(x)= & 16 t^{2}(t+1)^{2}\left(40 t^{2}-40 t+17\right)^{2}\left(120 t+40 t^{2}+97\right)^{2} \\
& \times\left(7,680,000 t^{8}+61,440,000 t^{7}+214,656,000 t^{6}+427,776,000 t^{5}\right. \\
& +534,691,200 t^{4}+433,804,800 t^{3}+225,855,440 t^{2}+69,477,280 t \\
& +9,866,003) \times p_{1}(t),
\end{aligned}
$$

where $p_{1}(t)$ is defined by (2.9), and $t=x+1 / 2>0$. Clearly, $S_{1}(x)>0$ for $x>-1 / 2$.

Similarly, we have

$$
S_{2}(x)=S_{3}(t)+t^{2} S_{4}(t),
$$

where

$$
\begin{aligned}
S_{3}(t)= & 19,544,408,064 t^{20}+195,444,080,640 t^{19}+\frac{4,351,725,010,944}{5} t^{18} \\
& +\frac{11,314,743,607,296}{5} t^{17}+\frac{94,415,373,656,064}{25} t^{16}+\frac{104,269,760,888,832}{25} t^{15}
\end{aligned}
$$




$$
\begin{array}{r}
+\frac{1,887,440,973,367,296}{625} t^{14}+\frac{888,059,638,659,072}{625} t^{13} \\
+\frac{1,921,294,271,712,768}{3,125} t^{12}+\frac{1,795,217,217,788,928}{3,125} t^{11} \\
+\frac{8,617,726,188,296,736}{15,625} t^{10}+\frac{928,558,757,330,976}{3,125} t^{9} \\
+\frac{1,217,964,858,530,932}{15,625} t^{8}+\frac{353,703,953,859,088}{15,625} t^{7} \\
+\frac{3,731,661,240,517,347}{156,250} t^{6}+\frac{151,574,123,955,363,957}{156,250,000} t \\
+\frac{20,166,045,810,484,915,467}{100,000,000,000}, \\
S_{4}(t)= \\
\quad-\frac{25,496,705,911,425,721}{156,250} t^{3}-\frac{78,452,580,264,405,241}{31,250,000} t^{2} \\
-
\end{array}
$$

It is clear that $S_{3}(t)>0$ for $t=x+1 / 2>0$. To prove that $S_{2}(x)>0$ for $x>-1 / 2$, it suffices to prove that $S_{4}(t)>0$ for $t>0$. In fact, it is easy to check that

$$
\begin{aligned}
S_{4}^{\prime}(t)= & \frac{4,490,117,734,277,163}{156,250} t^{2}-\frac{78,452,580,264,405,241}{15,625,000} t \\
& -\frac{25,060,239,724,662,741}{15,625,000}
\end{aligned}
$$

has a positive zero point $t_{0} \in(1 / 3,1 / 2)$ such that $S_{4}^{\prime}(t)<0$ for $t \in\left(0, t_{0}\right)$ and $S_{4}^{\prime}(t)<0$ for $t \in\left(t_{0}, \infty\right)$. Since $S_{4}(0), S_{4}(\infty)>0$ and

$$
\begin{aligned}
S_{4}\left(t_{0}\right)= & \frac{1,496,705,911,425,721}{156,250} t_{0}^{3}+\frac{11,608,440,209,633,547}{9,765,625} \\
& -\left(\frac{78,452,580,264,405,241}{31,250,000} t_{0}^{2}+\frac{25,060,239,724,662,741}{15,625,000} t_{0}\right) \\
> & \frac{1,496,705,911,425,721}{156,250}\left(\frac{1}{3}\right)^{3}+\frac{11,608,440,209,633,547}{9,765,625} \\
& -\left(\frac{78,452,580,264,405,241}{31,250,000}\left(\frac{1}{2}\right)^{2}+\frac{25,060,239,724,662,741}{15,625,000} \frac{1}{2}\right) \\
= & \frac{1,922,580,540,937,065,541}{16,875,000,000}>0,
\end{aligned}
$$

so we get $S_{4}(t)>0$ for $t>0$, which proves that $S_{2}(x)>0$ for $x>-1 / 2$ and completes the proof.

\section{Monotonicity and convexity}

In this section, we state and prove Theorems 1-3 on the monotonicity and convexity of three important functions $f_{1}(x), f_{2}(x)$, and $f_{3}(x)$ concerning the psi function, respectively. 
Theorem 1 The function

$$
x \mapsto f_{1}(x)=\exp \left(\psi\left(x+\frac{1}{2}\right)-\frac{1}{24} \frac{1}{x^{2}+7 / 40}\right)-x
$$

is decreasing from $(-1 / 2, \infty)$ onto $(0,1 / 2)$ and convex on $(-1 / 2, \infty)$.

Proof With (2.6) in hand, $f_{1}(x)$ can be written as

$$
f_{1}(x)=e^{\psi(x+1 / 2)-u(x)}-x
$$

Differentiation of this formula yields

$$
\begin{aligned}
f_{1}^{\prime}(x) & =\left(\psi^{\prime}(x+1 / 2)-u^{\prime}(x)\right) e^{\psi(x+1 / 2)-u(x)}-1, \\
f_{1}^{\prime \prime}(x) & =\frac{\psi^{\prime \prime}(x+1 / 2)-u^{\prime \prime}(x)+\left(\psi^{\prime}(x+1 / 2)-u^{\prime}(x)\right)^{2}}{e^{-\psi(x+1 / 2)+u(x)}} \\
& :=\frac{g(x)}{e^{-\psi(x+1 / 2)+u(x)}} .
\end{aligned}
$$

By (2.1) this yields

$$
\begin{aligned}
g(x+1) & -g(x) \\
= & {\left[\psi(x+3 / 2,2)-u^{\prime \prime}(x+1)\right]+\left[\psi(x+3 / 2,1)-u^{\prime}(x+1)\right]^{2} } \\
& -\left\{\psi(x+1 / 2,2)-u^{\prime \prime}(x)+\left[\psi(x+1 / 2,1)-u^{\prime}(x)\right]^{2}\right\} \\
= & p(x)\left[2 \psi(x+1 / 2,1)+r(x)+\frac{q(x)}{p(x)}\right] \\
:= & p(x) h(x),
\end{aligned}
$$

where $p(x), q(x), r(x)$ are defined by (2.7), (2.12), (2.13), respectively.

Similarly, we get

$$
h(x+1)-h(x)=-\frac{2}{(x+1 / 2)^{2}}+r(x+1)+\frac{q(x+1)}{p(x+1)}-r(x)-\frac{q(x)}{p(x)}=S(x) .
$$

By Lemma 6 we have $h(x+1)-h(x)<0$. It follows from $\lim _{x \rightarrow \infty} h(x)=0$ and Lemma 3 that $h(x)>\lim _{x \rightarrow \infty} h(x)=0$. Thanks to inequality (3.3), $p(x)<0$, and Lemma 5 , it follows that

$$
g(x+1)-g(x)=p(x) h(x)<0,
$$

which implies by Lemma 3 that $g(x)>\lim _{x \rightarrow \infty} g(x)=0$. Thus, in combination with (3.2), this leads to $f_{1}^{\prime \prime}(x)>0$, that is, $f_{1}$ is convex on $(-1 / 2, \infty)$, and $f_{1}^{\prime}$ is increasing on $(-1 / 2, \infty)$. Utilizing the asymptotic formulas (2.2)-(2.3), this yields

$$
\lim _{x \rightarrow \infty} f_{1}^{\prime}(x)=\lim _{x \rightarrow \infty} f_{1}(x)=0
$$


Therefore, we get that $f_{1}^{\prime}(x)<\lim _{x \rightarrow \infty} f_{1}^{\prime}(x)=0$, which implies that $f_{1}(x)$ is decreasing on $(-1 / 2, \infty)$. Moreover, we conclude that

$$
0=\lim _{x \rightarrow \infty} f_{1}(x)<f_{1}(x)<\lim _{x \rightarrow-1 / 2^{+}} f_{1}(x)=\frac{1}{2},
$$

which completes the proof.

\section{Theorem 2 The function}

$$
x \mapsto f_{2}(x)=\exp \psi\left(x+\frac{1}{2}\right)-x \exp \left(\frac{1}{24} \frac{1}{x^{2}+7 / 40}\right)
$$

is decreasing from $(0, \infty)$ onto $\left(0, e^{-\gamma / 4)}\right.$ and convex on $(1 / 2, \infty)$.

Proof We have

$$
\begin{aligned}
f_{2}(x) & =\exp \left(\frac{1}{24} \frac{1}{x^{2}+7 / 40}\right)\left\{\exp \left[\psi\left(x+\frac{1}{2}\right)-\frac{1}{24} \frac{1}{x^{2}+7 / 40}\right]-x\right\} \\
& =\exp \left(\frac{1}{24} \frac{1}{x^{2}+7 / 40}\right) f_{1}(x):=f_{0}(x) f_{1}(x) .
\end{aligned}
$$

Noting that

$$
\begin{aligned}
& f_{0}^{\prime}(x)=-\frac{1}{12} \frac{x}{\left(x^{2}+7 / 40\right)^{2}} \exp \left(\frac{1}{24} \frac{1}{x^{2}+7 / 40}\right) \leq 0 \text { for } x \geq 0, \\
& f_{0}^{\prime \prime}(x)=\frac{1}{57,600} \frac{14,400 x^{4}+2,080 x^{2}-147}{\left(x^{2}+7 / 40\right)^{4}} \exp \left(\frac{1}{24} \frac{1}{x^{2}+7 / 40}\right)>0 \quad \text { for } x \geq \frac{1}{2},
\end{aligned}
$$

we have

$$
\begin{aligned}
& f_{2}^{\prime}(x)=f_{0}^{\prime}(x) f_{1}(x)+f_{0}(x) f_{1}^{\prime}(x)<0 \quad \text { for } x \geq 0, \\
& f_{2}^{\prime \prime}(x)=f_{0}^{\prime \prime}(x) f_{1}(x)+2 f_{0}^{\prime}(x) f_{1}^{\prime}(x)+f_{0}(x) f_{1}^{\prime \prime}(x)>0 \quad \text { for } x \geq \frac{1}{2} .
\end{aligned}
$$

A simple calculation leads to

$$
\lim _{x \rightarrow \infty} f_{2}(x)=\lim _{x \rightarrow \infty} \exp \left(\frac{1}{24} \frac{1}{x^{2}+7 / 40}\right) \lim _{x \rightarrow \infty} f_{1}(x)=0 \quad \text { and } \quad \lim _{x \rightarrow 0+} f_{2}(x)=\frac{1}{4} e^{-\gamma}
$$

which completes the proof.

Theorem 3 Let $a \geq 0$. Then the function

$$
x \mapsto f_{3}(x)=\psi\left(x+\frac{1}{2}\right)-\ln x-\frac{1}{24} \frac{1}{x^{2}+a}
$$

is decreasing and convex on $(0, \infty)$ if and only if $a \geq 7 / 40$. 
Proof The necessity is obvious; it follows from the inequality $\lim _{x \rightarrow \infty} x^{5} f_{3}^{\prime}(x) \leq 0$. Indeed, using the asymptotic formulas (2.2), we have

$$
\lim _{x \rightarrow \infty} x^{5} f_{3}^{\prime}(x)=\frac{7}{240}-\frac{1}{6} a \leq 0
$$

which yields $a \geq 7 / 40$.

We now are a position to prove the sufficiency. By differentiation we have

$$
\begin{aligned}
& f_{3}^{\prime}(x)=\psi^{\prime}\left(x+\frac{1}{2}\right)-\frac{1}{x}+\frac{1}{12} \frac{x}{\left(x^{2}+a\right)^{2}} \\
& f_{3}^{\prime \prime}(x)=\psi^{\prime \prime}\left(x+\frac{1}{2}\right)+\frac{1}{x^{2}}+\frac{1}{12} \frac{1}{\left(x^{2}+a\right)^{2}}-\frac{1}{3} \frac{x^{2}}{\left(x^{2}+a\right)^{3}}
\end{aligned}
$$

Using (2.1), we have

$$
\begin{aligned}
f_{3}^{\prime \prime}(x+1)-f_{3}^{\prime \prime}(x)= & \psi^{\prime \prime}\left(x+\frac{3}{2}\right)+\frac{1}{(x+1)^{2}}+\frac{1}{12} \frac{1}{\left((x+1)^{2}+a\right)^{2}}-\frac{1}{3} \frac{x^{2}}{\left((x+1)^{2}+a\right)^{3}} \\
& -\psi^{\prime \prime}\left(x+\frac{1}{2}\right)-\frac{1}{x^{2}}-\frac{1}{12} \frac{1}{\left(x^{2}+a\right)^{2}}+\frac{1}{3} \frac{x^{2}}{\left(x^{2}+a\right)^{3}} \\
= & \frac{2}{(x+1 / 2)^{3}}+\frac{1}{(x+1)^{2}}+\frac{1}{12} \frac{1}{\left((x+1)^{2}+a\right)^{2}} \\
& -\frac{1}{3} \frac{x^{2}}{\left((x+1)^{2}+a\right)^{3}}-\frac{1}{x^{2}}-\frac{1}{12} \frac{1}{\left(x^{2}+a\right)^{2}}+\frac{1}{3} \frac{x^{2}}{\left(x^{2}+a\right)^{3}} \\
= & -\frac{P(x)}{12 x^{2}(2 x+1)^{3}\left(x^{2}+a\right)^{3}(x+1)^{2}\left(x^{2}+2 x+a+1\right)^{3}},
\end{aligned}
$$

where

$$
\begin{aligned}
P(x)= & 12(40 a-7) x^{12}+72(40 a-7) x^{11}+\left(1,536 a^{2}+7,576 a-1,290\right) x^{10} \\
& +\left(7,680 a^{2}+11,480 a-1,830\right) x^{9}+\left(2,016 a^{3}+16,788 a^{2}+11,038 a-1,563\right) x^{8} \\
& +\left(8,064 a^{3}+21,072 a^{2}+6,952 a-816\right) x^{7} \\
& +\left(1,440 a^{4}+14,112 a^{3}+16,710 a^{2}+2,840 a-252\right) x^{6} \\
& +\left(4,320 a^{4}+14,112 a^{3}+8,634 a^{2}+724 a-42\right) x^{5} \\
& +\left(576 a^{5}+5,652 a^{4}+8,862 a^{3}+2,853 a^{2}+115 a-3\right) x^{4} \\
& +\left(1,152 a^{5}+4,104 a^{4}+3,612 a^{3}+540 a^{2}+14 a\right) x^{3} \\
& +\left(96 a^{6}+936 a^{5}+1,764 a^{4}+966 a^{3}+39 a^{2}+a\right) x^{2} \\
& +\left(96 a^{6}+360 a^{5}+432 a^{4}+168 a^{3}\right) x+\left(12 a^{6}+36 a^{5}+36 a^{4}+12 a^{3}\right) .
\end{aligned}
$$

It is easy to check that the coefficients of the polynomial $P(x)$ are nonnegative for $a \geq 7 / 40$. Then we have $f_{3}^{\prime \prime}(x+1)-f_{3}^{\prime \prime}(x)<0$ for $x>0$. By Lemma 3 we get that $f_{3}^{\prime \prime}(x)>$ $\lim _{x \rightarrow \infty} f_{3}^{\prime \prime}(x)=0$, which implies that $f_{3}^{\prime}(x)$ is increasing on $(0, \infty)$. Therefore, $f_{3}^{\prime}(x)<$ $\lim _{x \rightarrow \infty} f_{3}^{\prime}(x)=0$, which completes our proof. 


\section{Inequalities for the psi function and harmonic number}

Denote $F_{i}(x)=f_{i}(x+1 / 2)(i=1,2,3)$ in Theorems 1 and 2 . Then, since $F_{1}$ is decreasing, $F_{1}(0)=e^{-\gamma-5 / 51}$, and $\lim _{x \rightarrow \infty} F(x)=1 / 2$, we get the following conclusion.

\section{Corollary 1}

(i) For $x>-1 / 2$, we have

$$
\psi(x+1)>\ln \left(x+\frac{1}{2}\right)+\frac{1}{24} \frac{1}{x^{2}+x+17 / 40} .
$$

(ii) For $x \geq 0$, we have

$$
\ln \left(x+\frac{1}{2}\right)+\frac{1}{24} \frac{1}{x^{2}+x+17 / 40}<\psi(x+1)<\ln \left(x+\alpha_{0}\right)+\frac{1}{24} \frac{1}{x^{2}+x+17 / 40}
$$

where the constants $1 / 2$ and $\alpha_{0}=e^{-\gamma-5 / 51} \approx 0.50903$ are the best possible.

Remark 1 Comparing (4.1) with (1.4), we find that, for $x>0$,

$$
\begin{aligned}
\ln \left(x+\frac{1}{2}\right) & <\ln \left(x+\frac{1}{2}\right)+\frac{1}{24} \frac{1}{x^{2}+x+17 / 40}<\psi(x+1) \\
& <\ln \left(x+\alpha_{0}\right)+\frac{1}{24} \frac{1}{x^{2}+x+17 / 40}<\ln \left(x+e^{-\gamma}\right) .
\end{aligned}
$$

In fact, it suffices to show that the last inequality is valid for $x>0$. Let

$$
D_{1}(x)=\ln \left(x+\alpha_{0}\right)+\frac{1}{24} \frac{1}{x^{2}+x+17 / 40}<\ln \left(x+e^{-\gamma}\right) .
$$

By differentiation we have

$$
\begin{aligned}
D_{1}^{\prime}(x) & =\frac{1}{x+\alpha_{0}}-\frac{1}{24} \frac{2 x+1}{\left(x^{2}+x+17 / 40\right)^{2}}-\frac{1}{x+\beta} \\
& =\frac{D_{2}(x)}{3\left(x+\alpha_{0}\right)(x+\beta)\left(40 x^{2}+40 x+17\right)^{2}},
\end{aligned}
$$

where

$$
\begin{aligned}
D_{2}(x)= & 4,800\left(e^{-\gamma}-\alpha_{0}\right) x^{4}+400\left(24 e^{-\gamma}-24 \alpha_{0}-1\right) x^{3}-40\left(232 \alpha_{0}-212 e^{-\gamma}+5\right) x^{2} \\
& -40\left(107 \alpha_{0}-97 e^{-\gamma}+10 \alpha_{0} e^{-\gamma}\right) x-\left(867 \alpha_{0}-867 e^{-\gamma}+200 \alpha_{0} e^{-\gamma}\right) \\
:= & a_{4} x^{4}+a_{3} x^{3}+a_{2} x^{2}+a_{1} x+a_{0} .
\end{aligned}
$$

It is easy to check that $a_{4}, a_{3}>0$ and $a_{2}, a_{1}, a_{0}<0$. Then by Lemma 4 we see that there is $x_{0}>0$ such that $D_{2}(x)<0$ for $x \in\left(0, x_{0}\right)$ and $D_{2}(x)>0$ for $x \in\left(x_{0}, \infty\right)$. This indicates that $D_{1}$ is decreasing on $\left(0, x_{0}\right)$ and increasing on $\left(x_{0}, \infty\right)$. Therefore, we conclude that, for $x>0$,

$$
D_{1}(x)<\max \left(D_{1}(0), D_{1}(\infty)\right)=0
$$


Remark 2 Similarly, we get the following inequalities:

$$
\begin{aligned}
\frac{1}{2} \ln \left(x^{2}+x+e^{-2 \gamma}\right) & <\ln \left(x+\frac{1}{2}\right)+\frac{1}{24} \frac{1}{x^{2}+x+17 / 40}<\psi(x+1) \\
& <\frac{1}{2} \ln \left(x^{2}+x+\frac{1}{3}\right)<\ln \left(x+\alpha_{0}\right)+\frac{1}{24} \frac{1}{x^{2}+x+17 / 40}
\end{aligned}
$$

for $x>0$. A direct computation shows that

$$
\begin{aligned}
& \lim _{x \rightarrow \infty} \frac{\psi(x+1)-\frac{1}{2} \ln \left(x^{2}+x+\frac{1}{3}\right)}{x^{-4}}=-\frac{1}{180}, \\
& \lim _{x \rightarrow \infty} \frac{\psi(x+1)-\ln \left(x+\frac{1}{2}\right)-\frac{1}{24} \frac{1}{x^{2}+x+17 / 40}}{x^{-6}}=\frac{2,071}{806,400},
\end{aligned}
$$

which implies that the approximation formula of the psi function given in (4.1) is superior to (1.6).

Since $F_{1}(1)=e^{286 / 291-\gamma}$, by the relation $\psi(n+1)=H_{n}-\gamma$ we deduce the following:

Corollary 2 For $n \in \mathbb{N}$, we have

$$
\gamma+\frac{1}{24} \frac{1}{n^{2}+n+17 / 40}+\ln (n+1 / 2)<H_{n}<\gamma+\frac{1}{24} \frac{1}{n^{2}+n+17 / 40}+\ln \left(n+\alpha_{1}\right),
$$

where $1 / 2$ and $\alpha_{1}=e^{286 / 291-\gamma} \approx 0.50021$ are the best possible.

Since $F_{2}$ is decreasing on $(-1 / 2, \infty), \lim _{x \rightarrow \infty} F_{2}(x)=0$, and

$$
F_{2}(0)=e^{-\gamma}-\frac{1}{2} e^{5 / 51}=\frac{1}{2} \beta_{0}, \quad F_{2}(1)=e^{1-\gamma}-\frac{3}{2} e^{5 / 291}=\frac{1}{2} \beta_{1},
$$

we get the following:

Corollary 3 For $x \geq 0$, we have

$$
\begin{aligned}
\ln \left(x+\frac{1}{2}\right)+\frac{1}{24} \frac{1}{x^{2}+x+17 / 40}< & \psi(x+1)<\ln \left(x+\frac{1}{2}\right)+\frac{1}{24} \frac{1}{x^{2}+x+17 / 40} \\
& +\ln \left[1+\frac{\beta_{0}}{2 x+1} \exp \left(-\frac{1}{24} \frac{1}{x^{2}+x+17 / 40}\right)\right]
\end{aligned}
$$

where $\beta_{0}=2 e^{-\gamma}-e^{5 / 51} \approx 0.019913$ is the best constant.

Corollary 4 For $n \in \mathbb{N}$, we have

$$
\begin{aligned}
\gamma+\ln \left(n+\frac{1}{2}\right)+\frac{1}{24} \frac{1}{n^{2}+n+17 / 40}< & H_{n}<\gamma+\ln \left(n+\frac{1}{2}\right)+\frac{1}{24} \frac{1}{n^{2}+n+17 / 40} \\
+ & \ln \left[1+\frac{\beta_{1}}{2 n+1} \exp \left(-\frac{1}{24} \frac{1}{n^{2}+n+17 / 40}\right)\right],
\end{aligned}
$$

where $\beta_{1}=2 e^{1-\gamma}-3 e^{5 / 291} \approx 0.00041845$ is the best constant. 
For $a=7 / 40$, since $F_{3}$ is decreasing on $(-1 / 2, \infty), \lim _{x \rightarrow \infty} F_{3}(x)=0$, and

$$
F_{3}(0)=\ln 2-\frac{5}{51}-\gamma=\delta_{0}, \quad F_{3}(1)=\frac{286}{291}-\ln \frac{3}{2}-\gamma=\delta_{1},
$$

we deduce the following:

Corollary 5 For $x \geq 0$, we have

$$
\ln \left(x+\frac{1}{2}\right)+\frac{1}{24} \frac{1}{x^{2}+x+17 / 40}+\delta_{0}^{*}<\psi(x+1)<\ln \left(x+\frac{1}{2}\right)+\frac{1}{24} \frac{1}{x^{2}+x+17 / 40}+\delta_{0}
$$

where $\delta_{0}^{*}=0$ and $\delta_{0}=\ln 2-5 / 51-\gamma \approx 0.017892$ are the best constants.

Corollary 6 For $n \in \mathbb{N}$, we have

$$
\gamma+\ln \left(n+\frac{1}{2}\right)+\frac{1}{24} \frac{1}{n^{2}+n+17 / 40}+\delta_{1}^{*}<H_{n}<\gamma+\ln \left(n+\frac{1}{2}\right)+\frac{1}{24} \frac{1}{n^{2}+n+17 / 40}+\delta_{1}
$$

where $\delta_{1}^{*}=0$ and $\delta_{1}=286 / 291-\ln (3 / 2)-\gamma \approx 0.00013710$ are the best constants.

From Theorem 1 we can obtain the following Batir-type inequalities for the psi function and harmonic number.

Corollary 7 For $x \geq 0$, we have

$$
\frac{1}{24\left(x^{2}+x+17 / 40\right)}-\ln \left(e^{Q(x)}-1\right)+c_{0}<\psi(x+1)<\frac{1}{24\left(x^{2}+x+17 / 40\right)}-\ln \left(e^{Q(x)}-1\right)+c_{0}^{*}
$$

with the best constants $c_{0}=\ln \left(e^{5,347 / 4,947}-1\right)-5 / 51-\gamma \approx-0.0088601$ and $c_{0}^{*}=0$, where

$$
Q(x)=\frac{4,800 x^{4}+19,200 x^{3}+28,480 x^{2}+18560 x+5,347}{3(x+1)\left(40 x^{2}+40 x+17\right)\left(40 x^{2}+120 x+97\right)} .
$$

Proof Let $G_{1}(x)=F_{1}(x+1)-F_{1}(x)=f_{1}(x+3 / 2)-f_{1}(x+1 / 2)$. Since $f_{1}$ is convex on $(-1 / 2, \infty)$, we have

$$
G_{1}^{\prime}(x)=f_{1}^{\prime}(x+3 / 2)-f_{1}^{\prime}(x+1 / 2)=f_{1}^{\prime \prime}(x+1 / 2+\theta)>0,
$$

which means that $G_{1}$ is increasing on $(0, \infty)$. Considering

$$
\begin{aligned}
G_{1}(x)= & \exp \left(\psi(x+2)-\frac{1}{24} \frac{1}{(x+3 / 2)^{2}+7 / 40}\right) \\
& -\exp \left(\psi(x+1)-\frac{1}{24} \frac{1}{(x+1 / 2)^{2}+7 / 40}\right)-1 \\
= & \exp \left(\psi(x+1)-\frac{1}{24} \frac{1}{(x+1 / 2)^{2}+7 / 40}\right)\left(e^{Q(x)}-1\right)-1 \\
G_{1}(0)= & \exp \left(\frac{286}{291}-\gamma\right)-e\left(-\frac{5}{51}-\gamma\right)-1 \text { and } \lim _{x \rightarrow \infty} G_{1}(x)=0
\end{aligned}
$$


we have

$$
G_{1}(0)<\exp \left(\psi(x+1)-\frac{1}{24} \frac{1}{(x+1 / 2)^{2}+7 / 40}\right)\left(e^{Q(x)}-1\right)-1<0
$$

which attains the desired inequality.

The increasing property of $G_{1}$ and

$$
G_{1}(1)=\exp \left(\frac{2,303}{1,542}-\gamma\right)-\exp \left(\frac{286}{291}-\gamma\right)-1
$$

yield a sharp bound of harmonic number.

Corollary 8 For $n \in \mathbb{N}$, we have

$$
\begin{aligned}
\gamma & +\frac{1}{24\left(n^{2}+n+17 / 40\right)}-\ln \left(e^{Q(n)}-1\right)+c_{1} \\
& <H_{n}<\gamma+\frac{1}{24\left(n^{2}+n+17 / 40\right)}-\ln \left(e^{Q(n)}-1\right)+c_{1}^{*},
\end{aligned}
$$

where $c_{1}=286 / 291-\gamma+\ln \left(e^{76,387 / 149,574}-1\right) \approx-0.00018438$ and $c_{1}^{*}=0$ are the best constants.

Remark 3 Note that since $\psi(n+1)=H_{n}-\gamma, G_{1}(n)$ is written as

$$
\begin{aligned}
G_{1}(n)= & \exp \left(H_{n+1}-\gamma-\frac{1}{24} \frac{1}{(n+3 / 2)^{2}+7 / 40}\right) \\
& -\exp \left(H_{n}-\gamma-\frac{1}{24} \frac{1}{(n+1 / 2)^{2}+7 / 40}\right)-1 .
\end{aligned}
$$

Then by $G_{1}(1) \leq G_{1}(n)<G_{1}(\infty)$ we have the following Alzer-type inequalities:

$$
1.7807 \approx \exp \left(\frac{2,303}{1,542}\right)-\exp \left(\frac{286}{291}\right)<e^{H_{n+1}-u_{n+1}}-e^{H_{n}-u_{n}}<e^{\gamma} \approx 1.7811,
$$

where

$$
u_{n}=\frac{1}{24} \frac{1}{(n+1 / 2)^{2}+7 / 40} .
$$

Remark 4 Similarly, it is easy to check that

$$
G_{2}(x)=F_{2}(x+1)-F_{2}(x)=f_{2}(x+3 / 2)-f_{2}(x+1 / 2)
$$

is increasing on $(0, \infty)$. Then, from

$$
-0.00018779 \approx \frac{3}{2} e^{5 / 291}-\frac{5}{2} e^{5 / 771}+e^{1-\gamma}\left(e^{1 / 2}-1\right)=G_{2}(1) \leq G_{2}(n)<G_{2}(\infty)=0
$$


we derive other Alzer-type inequalities:

$$
\left(n+\frac{3}{2}\right) u_{n+1}-\left(n+\frac{1}{2}\right) u_{n}+d_{1}<e^{H_{n+1}}-e^{H_{n}}<\left(n+\frac{3}{2}\right) u_{n+1}-\left(n+\frac{1}{2}\right) u_{n}+d_{0},
$$

where $d_{0}=0$ and $d_{1}=3 e^{5 / 291} / 2-5 e^{5 / 771} / 2+e^{1-\gamma}\left(e^{1 / 2}-1\right) \approx-0.00018779$ are the best constants with $u_{n}$ as in (4.4).

Using the increasing property of $F_{1}^{\prime}$, and noting that $F_{1}^{\prime}\left(-1^{+}\right)=-1, F_{1}^{\prime}(0)=\left(\pi^{2} / 6+\right.$ $200 / 867) e^{-\gamma-5 / 51}-1$, and $F_{1}(\infty)=0$, we get

$$
\left(\frac{1}{6} \pi^{2}+\frac{200}{867}\right) e^{-\gamma-5 / 51}-1<\left(\psi^{\prime}(x+1)-u^{\prime}(x)\right) e^{\psi(x+1)-u(x)}-1<0,
$$

which implies the following:

\section{Corollary 9}

(i) For $x>-1$, we have

$$
\psi^{\prime}(x+1)<-\frac{1}{12} \frac{x+1 / 2}{\left(x^{2}+x+17 / 40\right)^{2}}+\exp \left(-\psi(x+1)+\frac{1}{24\left(x^{2}+x+17 / 40\right)}\right) .
$$

(ii) For $x \geq 0$, we have the double inequalities

$$
\begin{aligned}
-\frac{1}{12} & \frac{x+1 / 2}{\left(x^{2}+x+17 / 40\right)^{2}}+\lambda_{1} \exp \left(-\psi(x+1)+\frac{1}{24\left(x^{2}+x+17 / 40\right)}\right) \\
< & \psi^{\prime}(x+1)<-\frac{1}{12} \frac{x+1 / 2}{\left(x^{2}+x+17 / 40\right)^{2}} \\
& +\lambda_{2} \exp \left(-\psi(x+1)+\frac{1}{24\left(x^{2}+x+17 / 40\right)}\right),
\end{aligned}
$$

where $\lambda_{1}=\left(\pi^{2} / 6+200 / 867\right) e^{-\gamma-5 / 51} \approx 0.95474$ and $\lambda_{2}=1$ are the best constants.

Remark 5 Elezovic et al. [32] proved the inequality

$$
\psi^{\prime}(x)<e^{-\psi(x)}
$$

for $x>0$. It has been improved by Batir [22] as

$$
\left(x+a^{*}\right) e^{-2 \psi(x)}<\psi^{\prime}(x+1)<\left(x+b^{*}\right) e^{-2 \psi(x)}
$$

for $x>0$ with the best constants $a^{*}=1 / 2$ and $b^{*}=\pi^{2} e^{-2 \gamma} / 6$. The last corollary gives another improvement of (4.5).

From the proof of Theorem 1 we see that $g(x)>0$ for $x>-1 / 2$, which can be written as the following corollary.

Corollary 10 For $x>0$, we have

$$
\psi^{\prime \prime}(x)-u^{\prime \prime}(x-1 / 2)+\left(\psi^{\prime}(x)-u^{\prime}(x-1 / 2)\right)^{2}>0,
$$

where $u(x)$ is defined by (2.6). 
Remark 6 Batir [23] showed that, for $x>0$,

$$
\psi^{\prime}(x)^{2}+\psi^{\prime \prime}(x)>0
$$

Therefore, inequality (4.6) can be written as

$$
\psi^{\prime \prime}(x)+\psi^{\prime}(x)^{2}>\Delta(x)
$$

where

$$
\begin{aligned}
\Delta(x)= & -\frac{400}{3} \frac{2 x-1}{\left(40 x^{2}-40 x+17\right)^{2}} \\
& \times\left(\psi^{\prime}(x)-\frac{14,400 x^{4}-28,800 x^{3}+22,880 x^{2}-8,480 x+1,073}{3(2 x-1)\left(40 x^{2}-40 x+17\right)^{2}}\right) .
\end{aligned}
$$

Indeed, this result is optimal due to

$$
\begin{aligned}
& \psi^{\prime \prime}(x)-\frac{400}{3} \frac{120(x-1 / 2)^{2}-7}{\left(40(x-1 / 2)^{2}+7\right)^{3}}+\left(\psi^{\prime}(x)+\frac{400}{3} \frac{(x-1 / 2)}{\left(40(x-1 / 2)^{2}+7\right)^{2}}\right)^{2} \\
& =\psi^{\prime \prime}(x)+\psi^{\prime}(x)^{2}-\frac{400}{3} \frac{1}{\left(40 x^{2}-40 x+17\right)^{2}} \Delta(x),
\end{aligned}
$$

where

$$
\Delta(x)=-(2 x-1) \psi^{\prime}(x)+\frac{14,400 x^{4}-28,800 x^{3}+22,880 x^{2}-8,480 x+1,073}{3\left(40 x^{2}-40 x+17\right)^{2}} .
$$

A numeric computation shows that $\Delta(x)>0$ for $0<x<2 / 5$ and $x>3 / 2$, and so inequality (4.6) is better than (4.7).

From the inequalities $f_{3}^{\prime}(x)<0$ and $f_{3}^{\prime \prime}(x)>0$ on $(0, \infty)$ for $a=7 / 40$, which are given in the proof of Theorem 3, we have the following:

Corollary 11 For $x>0$, we have the following inequalities:

$$
\begin{aligned}
& \psi^{\prime}\left(x+\frac{1}{2}\right)<\frac{1}{3} \frac{4,800 x^{4}+1,280 x^{2}+147}{x\left(40 x^{2}+7\right)^{2}}, \\
& \psi^{\prime \prime}\left(x+\frac{1}{2}\right)>-\frac{1}{3} \frac{192,000 x^{6}+52,800 x^{4}+20,440 x^{2}+1,029}{x^{2}\left(40 x^{2}+7\right)^{3}}, \\
& \psi^{\prime \prime \prime}\left(x+\frac{1}{2}\right)<2 \frac{2,560,000 x^{8}+512,000 x^{6}+694,400 x^{4}+54,880 x^{2}+2,401}{x^{3}\left(40 x^{2}+7\right)^{4}} .
\end{aligned}
$$

\section{Competing interests}

The authors declare that they have no competing interests.

\section{Authors' contributions}

All authors contributed equally to the writing of this paper. All authors read and approved the final manuscript.

\section{Author details}

'School of Mechanical, Electronic and Control Engineering, Beijing Jiaotong University, Beijing, 100044, China. ${ }^{2}$ Tangshan Railway Vehicle Co. LTD, Tangshan, 063035, China. ${ }^{3}$ Department of Mathematics, Beijing Jiaotong University, Beijing, 100044 , China. 


\section{Acknowledgements}

This paper was supported by the Natural Science Foundation of China under Grant 11371050. The authors would like to thank the referees for their valuable comments and suggestions which essentially improved the quality of this paper.

Received: 28 February 2016 Accepted: 19 May 2016 Published online: 10 June 2016

\section{References}

1. Chen, CP: Monotonicity properties of functions related to the psi function. Appl. Math. Comput. 217, 2905-2911 (2010)

2. Chu, YM, Zhang, XM, Tang, XM: An elementary inequality for psi function. Bull. Inst. Math. Acad. Sin. 3(3), 373-380 (2008)

3. Guo, BN, Qi, F: Some properties of the psi and polygamma functions. Hacet. J. Math. Stat. 39, $219-231$ (2010)

4. Lu, D: Some new convergent sequences and inequalities of Euler's constant. J. Math. Anal. Appl. 419, 541-552 (2014)

5. Mortici, C: Improved convergence towards generalized Euler-Mascheroni constant. Appl. Math. Comput. 215 3443-3448 (2010)

6. Mortici, C: Fast convergences towards Euler-Mascheroni constant. Comput. Appl. Math. 29, 479-491 (2010)

7. Mortici, C: A quicker convergence toward the gamma constant with the logarithm term involving the constant e. Carpath. J. Math. 26(1), 86-91 (2010)

8. Mortici, C, Chen, CP: On the harmonic number expansion by Ramanujan. J. Inequal. Appl. 2013, Article ID 222 (2013)

9. Qi, F, Cui, RQ, Chen, CP, Guo, RN: Some completely monotonic functions involving polygamma functions and an application. J. Math. Anal. Appl. 310, 303-308 (2005)

10. Sintǎmărian, A: A generalization of Euler's constant. Numer. Algorithms 46, 141-151 (2007)

11. Sintǎmǎrian, A: Some inequalities regarding a generalization of Euler's constant. J. Inequal. Pure Appl. Math. 9, 46 (2008)

12. Tims, SR, Tyrrell, JA: Approximate evaluation of Euler's constant. Math. Gaz. 55, 65-67 (1971)

13. Qi, F, Guo, BN: Sharp inequalities for the psi function and harmonic numbers. Available online at http://arxiv.org/abs/0902.2524

14. $\mathrm{Wu}, \mathrm{LL}, \mathrm{Chu}, \mathrm{YM}$ : An inequality for the psi functions. Appl. Math. Sci. 2(9-12), 545-550 (2008)

15. $\mathrm{Wu}, \mathrm{LL}, \mathrm{Chu}, \mathrm{YM}, \mathrm{Tang}, \mathrm{XM}$ : Inequalities for the generalized logarithmic mean and psi functions. Int. J. Pure Appl. Math. 48(1), 117-122 (2008)

16. Yang, ZH, Chu, YM: Sharp bounds for psi function. Appl. Math. Comput. 268, 1055-1063 (2015)

17. Zhang, XM, Chu, YM: An inequality involving the gamma function and the psi function. Int. J. Mod. Math. 3(1), 67-73 (2008)

18. Zhao, TH, Yang, ZH, Chu, YM: Monotonicity properties of a function involving the psi function with applications J. Inequal. Appl. 2015, Article ID 193 (2015)

19. DeTemple, DW, Wang, SH: Half integer approximations for the partial sums of the harmonic series. J. Math. Anal. Appl. $160,149-156(1991)$

20. Guo, BN, Qi, F: Sharp inequalities for the psi function and harmonic numbers. Analysis 34, 1-10 (2014)

21. Batir, N: Inequalities for the gamma function. RGMIA Res. Rep. Collect. 12, 9 (2009)

22. Batir, N: Sharp bounds for the psi function and harmonic numbers. Math. Inequal. Appl. 14, 917-925 (2011)

23. Batir, N: Some new inequalities for gamma and polygamma functions. J. Inequal. Pure Appl. Math. 6, 103 (2005)

24. Batir, N: On some properties of the digamma and polygamma functions. J. Math. Anal. Appl. 328, $452-465$ (2007)

25. Alzer, $\mathrm{H}$ : Sharp inequalities for the harmonic numbers. Expo. Math. 24, 385-388 (2006)

26. Alzer, H: Inequalities for the harmonic numbers. Math. Z. 267, 367-384 (2011)

27. Villarino, MB: Ramanujan's harmonic number expansion into negative powers of triangular number. J. Inequal. Pure Appl. Math. 9, 89 (2008)

28. Yang, ZH, Chu, YM, Zhang, XH: Necessary and sufficient conditions for functions involving the psi function to be completely monotonic. J. Inequal. Appl. 2015, Article ID 157 (2015)

29. Abramowitz, M, Stegun, IA: Handbook of Mathematical Functions with Formulas, Graphs, and Mathematical Tables, National Bureau of Standards, Washington, 9th printing edn. Applied Mathematics Series, vol. 55 (1970)

30. Elbert, A, Laforgia, A: On some properties of the gamma function. Proc. Am. Math. Soc. 128, 2667-2673 (2000)

31. Yang, ZH, Chu, YM, Tao, XJ: A double inequality for the trigamma function and its applications. Abstr. Appl. Anal. 2014, Article ID 702718 (2014)

32. Elezovic, N, Giordano, C, Pecaric, J: The best bounds for Gautschi's inequality. Math. Inequal. Appl. 3, 239-252 (2000) 